\section{The Nobel Prize in}

\section{Physics 2003}

Gianni Blatter and Vadim Geshkenbein, Theoretische Physik, ETH Zürich, Switzerland

$T$ he phenomenology of condensed quantum liquids has been the winning theme in last year's Nobel competition-Alexei A. Abrikosov (Argonne National Laboratory), Vitaly L. Ginzburg (Lebedev Institute in Moscow), and Anthony J. Leggett (University of Illinois at Urbana) share the 2003 Nobel prize in physics for their "pioneering contributions to the theory of superconductors and superfluidity" Their names are added to the illustrious list of Nobel laureates in the field of low temperature physics.

Superconductivity/fluidity has always been the most glamorous topic in condensed matter physics. The milestones characterizing the field start with the discovery of superconductivity in mercury by Kamerlingh Onnes in 1911. In 1938, the superfluid phase of ${ }^{4} \mathrm{He}$ was found by Kapitsa in Moscow and independently by Allen and Misener in Cambridge. Since metallic mercury transports electric current free of dissipation the original name super conductor' stuck; liquid He is uncharged and hence the dissipation-free mass flow is termed superfluidity. While the superfluid properties of bosonic ${ }^{4} \mathrm{He}$ were quickly understood in terms of a condensation into a macroscopic quantum state (Landau, 1941), the microscopic origin of superconductivity remained a puzzle over hair a century, until Bardeen, Cooper, âîd Schrieffer (1957) prōposed a pairing mechanism allowing bound fermions to condense. This paved the way for the theoretical prediction that fermionic ${ }^{3} \mathrm{He}$ should become superfluid as well, but it took another decade until Lee, Osheroff, and Richardson observed the superfluid phases of ${ }^{3} \mathrm{He}$ in 1972. The unexpected discovery of high-temperature superconductors by Bednorz and Müller in 1986 defined the most prominent research direction in condensed matter physics for the decade to follow. Last year the spectacular insights that follow from phenomenological theories have been properly recognized.

\section{The Ginzburg-Landau Theory}

Upon cooling to sufficiently low temperatures, electrons or atoms must lose entropy and organize themselves into a more ordered state. In doing so, the system undergoes a reduction of symmetry, the central idea of Landau's phenomenological theory of phase transitions dating back to 1937 . The symmetry breaking is captured by an order parameter, whose nature is most obvious in a ferromagnet where it quantifies the material's magnetization. Also, the idea of Bose-Einstein condensation into a macroscopically occupied quantum state suggests a superfluid order parameter in the form of a complex wave function $\Psi$. Because of the Pauli principle, it remained completely unclear, however, what could be a proper order parameter for the fermionic electron system in a superconducting metal and it required the remarkable intuition of Ginzburg and Landau to propose an order parameter in the form of a complex electronic wavefunction $\Psi^{\prime}=\sqrt{\rho} \exp (i \varphi)$. The Ginzburg-Landau free energy functional, which they constructed based on this order parameter set the foundation for the complete phenomenological understanding of superconducting materials.

Quite astonishingly, the rich phenomenology of the superconducting state could be quantitatively described without knowledge of the underlying microscopic mechanism responsible for the condensation in the first place. Early work of Gorter, Casimir, and the London brothers, explained numerous thermodynamic and electromagnetic aspects of the superconducting state. In particular, the London equation relating the superconducting current $j_{s}$ to the vector potential $\mathbf{A}$ via the superconducting density $\rho_{\mathrm{s}}, \mathbf{j}_{\mathrm{s}}=\rho_{\mathrm{s}} \mathbf{A}$, properly explained the Meissner-Ochsenfeld effect, the complete expulsion of a magnetic field from the interior of a superconductor. However, discrepancies with experiment remained, particularly in films subject to a parallel magnetic field where the expression for the critical field $H_{c \|}$ destroying superconductivity failed. Furthermore, the London theory was unable to provide a positive surface energy for the superconducting-normal interface, hence a basic ingredient to Landau's intermediate state (1937) remained unexplained. These practical shortcomings were overcome in the 1950 paper of Ginzburg and Landau. In setting up their famous energy functional they allowed for the presence of a magnetic field. The minimal gauge invariant coupling $|(-i \hbar \nabla-(e / c) \mathbf{A}) \Psi|^{2}$ led them to the subtle remark that ' $e$ is a charge, which there is no reason to consider as different from the electronic charge', thus accepting the possibility that it may take a different value. The ability to incorporate variations in the $\Psi$-field due to boundary conditions, external magnetic fields, and currents, allowed them to remedy the deficiencies of the London theory. With the Ginzburg-Landau energy functional, the vector potential $\mathbf{A}(\mathbf{r})$ properly described within the London theory got its partner field, the macroscopic wave function $\Psi(\mathbf{r})$, thus providing the complete phenomenological description of a charged quantum fluid. However, it still required Gorkov's derivation from the microscopic BCS theory (1959) until the Ginzburg-Landau equations acquired their definitive status with an effective charge $2 \mathrm{e}$.

Virith the appearance of a second ('Y') fieid a new dirnensioniess parameter comparing the relative 'stiffness' of the two fields enters the theory: the Ginzburg-Landau parameter $\kappa=\lambda / \xi$ measures the ratio in lengths associated with changes in the electromagnetic response (the London penetration depth $\lambda$ ) and in the superconducting density (the coherence length $\xi$ ). The limiting value $\kappa=1 / \sqrt{2}$ separating superconductors with positive surface energy $(\kappa<1 / \sqrt{2})$ from those with negative surface energy at large $\kappa>1 / \sqrt{2}$, was properly identified and the instability associated with the negative surface energy was noted, but the consequences were not pursued. At the time, superconductivity had been observed in elementary superconductors characterized by a small $\kappa$, hence Ginzburg and Landau decided that 'since from experimental data it follows that $\kappa \ll 1 \ldots$ another limiting case when $\kappa \rightarrow \infty$ does not offer any intrinsic interest (and) we shall not discuss it'.

\section{Type II Superconductors}

It remained for Alexei Abrikosov to uncover the scientific gold mine that opened up with the 'superconductors of the second group' involving large $\kappa$, nowadays called type II superconductors. Abrikosov's first contact with these 'unconventional' superconductors dates back to the early fifties, when Nikolay Zavaritskii changed the fabrication mode of his thin Sn and Tl films, keeping the substrate at low temperatures in order to improve their homogeneity. While the behavior of his previous room temperature evaporated films was in good agreement with theory, the new ones deviated strongly. This led Abrikosov and Zavaritskii to propose that these new films belong to the 'second group' of superconductors with $\kappa>1 / \sqrt{2}$ and indeed their critical field matched well the theoretical result found by Abrikosov in 1952. The breakthrough came in 1957, when Abrikosov obtained the basic phenomenological description of type II superconductors in a single stroke. The instability noticed 


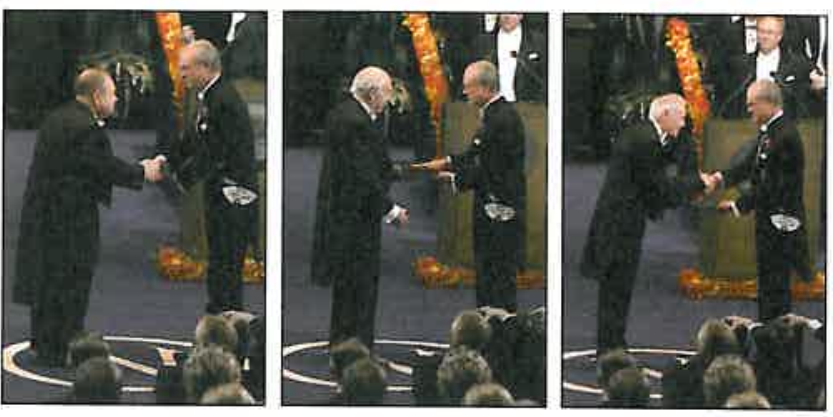

- Photographs: From left to right:Abrikosov, Ginzburg, and Leggett receiving the Nobel Prize in Physics 2003.

by Ginzburg and Landau allowed the magnetic field to enter the superconductor without destroying it. This leads to the appearance of an interesting new thermodynamic phase, the mixed or Shubnikov phase, where superconducting and normal regions peacefully coexist. The normal regions appear in the cores (of size $\xi$ ) of vortices binding individual magnetic flux quanta $\Phi_{0}=h c / 2 e$ on the scale $\lambda$, with the charge ' $2 e^{\prime}$ appearing in $\Phi_{0}$ a consequence of the pairing mechanism; since $\lambda>\xi$, the vortices repel and arrange in a stable lattice, nowadays named after its inventor Alexei Abrikosov. In his 1957 paper, Abrikosov derived the periodic vortex structure near the upper critical field $H_{c 2}$, where the superconductivity is totally suppressed, determined the magnetization $M(H)$, calculated the field $H_{c 1}$ of first penetration, analyzed the structure of individual vortex lines, found the structure of the vortex lattice at low fields, and compared his findings with experiments on what appeared to be the first observation of this novel mixed state, the $B(H)$ curves measured on $\mathrm{Pb}$ alloys by Shubnikov, Khotkevich, Shepelev, and Riabinin in 1937. Although this already makes an impressive list, the phenomenology of type II superconductors continued to develop for many years. It turns out that all technologically useful superconductors are 'of the second group'; these materials smoothly incorporate the large magnetic fields appearing in most technological applications, hence they 'bend' rather than 'break' under the action of fields and currents. In particular, the cuprate high temperature superconductors (discovered by Bednorz and Müller in 1986) are strong type II materials exhibiting an amazing variety of vortex phases with novel properties. Meantime, the Abrikosov lattice has undergone a 'metamorphosis' to the field of Vortex Matter, the filigrane arrangements formed by vortex-lines residing within conventional matter made of atoms and electrons.

As so often in the field of superconductivity it took many years to understand the rich and complex phenomenology of these materials. Political events also hampered the developments : in 1937 Shubnikov was accused of 'anti-Soviet activity' and sentenced to death; the same accusation led to the imprisonment of Landau in Moscow. Thus, Shubnikov's 1937 results on the mixed state failed to catch the attention of the community for 20 years. Similarly, the cold war hampered the diffusion of the amazing achievements of Russian theoreticians in the west.

\section{Superfluid ${ }^{3} \mathrm{He}$}

${ }^{3} \mathrm{He}$ is an uncharged quantum fluid with a repulsive interatomic interaction at short distances favoring pairing in a finite angular momentum state. The order parameter field then assumes a complex structure incorporating additional internal degrees of freedom: the unconventional pairing involves atoms with parallel spins (triplet channel with $S=1$ ) and orbital momentum $L=1$. As a result, the superfluid phase is anisotropic, with $\hat{\mathbf{d}}$ and $\hat{\mathbf{l}}$ two direc- tions associated with the additional spin- and orbital degrees of freedom. Hence cooling ${ }^{3} \mathrm{He}$ down to low temperatures, leads not only to the breaking of gauge symmetry but also of the rotational symmetry in spin- and orbital space, thus requiring a macroscopic wavefunction with a tensorial structure involving $3 \times 3$ complex components (at least in principle). In practice, the lowest energy state is isotropic, with the spin- and orbital angular momenta adding to zero (the BW state first found by Balian and Werthamer in 1963). Its excitation spectrum exhibits a constant energy gap just like a conventional BCS superconductor. Even earlier, Anderson and Morel had proposed an anisotropic state, with zero energy gap along the $\hat{\mathbf{l}}$-axis (the AM state). But another decade passed until Lee, Osheroff, and Richardson finally observed the superfluid phases of ${ }^{3} \mathrm{He}$ in 1972, an A-phase at elevated temperatures and pressures and a B-phase in the remainder of the low temperature phase diagram. It was Anthony Leggett who unravelled the puzzle of interpreting the experimental data with the correct theoretical picture. The NMR (nuclear magnetic resonance) data identifying the new phases exhibited sharp resonance frequencies, unexpectedly shifted in the A but not in the B phase, which Leggett explained in terms of a new'spontaneously broken spin-orbit symmetry'. While this symmetry is trivially broken in the AM state through selection of specific directions $\hat{\mathbf{d}}$ and $\hat{\mathbf{l}}$, the situation is more subtle for the isotropic BW state, where neither $\hat{\mathbf{d}}$ nor $\hat{\mathbf{l}}$ but only their relative orientation is fixed. This subtly broken rotational symmetry manifests itself only in the dynamical response. Soon after the discovery of the new superfluids, Leggett presented his explanation for the observed shift in terms of a dipole field enhanced by the macroscopic alignment of nuclear spins and predicted the existence of additional longitudinal modes in the AM and BW phases. He identified the AM state as a possible candidate for the observed A phase but noted the competition with the lower energy BW state. This led Anderson and Brinkman (1973) to reconsider the AM state and they demonstrated its stabilization due to the feedback effects of the condensation on the interparticle interactions (nowadays the ABM state carries the initials of all three authors). Finally, Ambegaokar and Mermin (1973) identified the third phase (termed A1) appearing at finite magnetic fields as the first magnetic superfluid.

In retrospect, the complexity of ${ }^{3} \mathrm{He}$ teaches us an interesting story. At first sight ${ }^{4} \mathrm{He}$ and ${ }^{3} \mathrm{He}$ are two fluids characterized by the same isotropic Lennard-Jones type interaction. The source of their difference lies in the absence of one neutron in the nucleus of a ${ }^{3} \mathrm{He}$ atom which is enclosed in a rigid electronic shell governing the interaction between atoms. Still, the low temperature properties of the two fluids are as different as one could imagine, owing to the quantum-mechanical properties of their light constituents. One may wonder what ingenuity it would take for an observer living at low temperatures and confronted with this complexity to guess the correct simple high-energy Hamiltonian responsible for this diversity of phases. This inverse task, guessing the correct "highenergy Hamiltonian" from observations at low energies, is analogous to the central problem facing the particle physics community. In condensed matter theory the program of deriving the correct microscopic Hamiltonian from experimental observations has been carried out many times, with the BCS Hamiltonian serving as a prominent example. One should appreciate, however, the amazing insights that phenomenological low-energy theories, such as those introduced and used by Ginzburg, Abrikosov, and Leggett, have contributed to our understanding of the world we live in.

\section{Acknowledgements}

We thank M. Rice, D. Vollhardt, and P. Wölfle for discussions. 